\title{
The US imaging in the management of abdominal aortic aneurysm: perspective
}

\author{
E Scarcello ${ }^{*}$, A Conti, M Mazzei, F Morrone, P Piro, S Tarsitano, P Perri, F Intrieri \\ From de Senectute: Age and Health Forum \\ Catanzaro, Italy. 5-7 December 2009
}

The development of surgical techniques depends on the concomitant development of imaging techniques. Imaging with ultrasonography (US), computed tomography (CT) and magnetic resonance imaging (MRI) is evolving at rapid pace.

The basic need for treatment is mainly important for the endovascular aortic repair (EVAR) that requires information about many different anatomical aspects regarding aneurysmal neck, renal arteries, and diameter of aneurysmal free lumen, aortic bifurcation, diameter and length of iliac vessels.

At present, the development of color-Doppler instruments is surely comparable with that of CT and MR imaging, and an important development has been made also for different types of ultrasonographic contrast medium. The intravascular US (IVUS) mode appears particularly important, that allows real-time detection of a lot of information useful in EVAR.

At present, in elective treatment US examination plays a role in different steps:

- diagnosis

- AAA classification (below, iuxta or suprarenal AAA)

- per operative management with IVUS mode [1]

- postoperative follow-up.

In emergency, the US examination has a place first of all as a bedside exam for patients with a suspected ruptured abdominal aortic aneurysm (rAAA). In haemodinamically stable patients the firtsline US examination appears mandatory during an examination of patients to detect the AAA and to avoid misdiagnosis or, in the opposite case, to abstain from undue X-rays exposition. Actually the physical examination allows the detection

Unit of Vascular and Endovascular Surgery, Azienda Ospedaliera di Cosenza, Italy of an AAA in about $43 \%$ [2] of the patients, and its sensibility results lower for an iliac aneurysm, whereas that of US is up to $99 \%$ [3]. In haemodinamically instable patients emergency US exam appears at present the only possible evaluation in the immediately preoperative stage, as the CT exam provides an obligatory and dangerous delay. Moreover in haemodinamically instable patients an emergency IVUS exam could integrate the angiography during endovascular treatment of rAAA to better detect needed information, ${ }^{1}$ but physicians, nurses, and technicians need to work through a learning curve in order to properly interpret the images generated and maximize the usefulness of the technology.

Hypothetically abdominal US through a friendlier learning curve could integrate the angiographic valuation to better define the outer diameter and the parietal features of the aortic neck, as the angiography does not reveal localization and extent of the thrombus and/or calcification.

Published: 19 May 2010

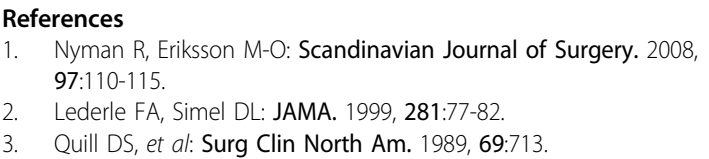

doi:10.1186/1471-2318-10-S1-L17

Cite this article as: Scarcello et al.: The US imaging in the management of abdominal aortic aneurysm: perspective. BMC Geriatrics 2010 10(Suppl 1):L17. 